\title{
Minimal Models of Frustrated Quantum Magnets
}

Cite as: AIP Conference Proceedings 816, 55 (2006); https://doi.org/10.1063/1.2178031 Published Online: 14 February 2006

\section{Frédéric Mila}

\section{ARTICLES YOU MAY BE INTERESTED IN}

Quantum criticality

Physics Today 64, 29 (2011); https://doi.org/10.1063/1.3554314

Geometrical frustration

Physics Today 59, 24 (2006); https://doi.org/10.1063/1.2186278

Do quantum spin liquids exist?

Physics Today 69, 30 (2016); https://doi.org/10.1063/PT.3.3266

\section{Conference Proceedings} Get $30 \%$ off all
print proceedings! Get $30 \%$ off all
print proceedings!

AIP
Enter Promotion Code PDF-30 at checkout

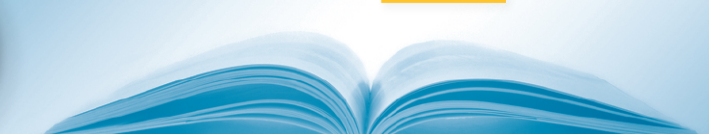




\title{
Minimal Models of Frustrated Quantum Magnets
}

\author{
Frédéric Mila \\ Institute of Theoretical Physics, Ecole Polytechnique Fédérale de Lausanne (EPFL), CH-1015 \\ Lausanne, Switzerland
}

\begin{abstract}
Frustrated quantum magnets are a real challenge to theorists because standard analytical and numerical methods fail in most cases. Significant progress has been recently achieved however on the basis of what one may call minimal models, i.e. models which keep at least one important aspect of frustration, but which are otherwise simple enough to be understood. In this paper, I will concentrate on two types of minimal models, Quantum Dimer Models and hard-core boson models. In the case of Quantum Dimer Models, I will review the evidence in favour of a Resonating Valence Bond (RVB) spin-liquid phase, and I will discuss under which circumstances such models might be good effective models. For hard-core boson models, which have been successfully used for quite some time for ladders and other non-frustrated models of coupled dimers, I will describe the specificities introduced by frustration (reduced kinetic energy, correlated hopping,...) and the unexpected properties they induce.
\end{abstract}

Keywords: Frustrated magnetism, quantum dimer models, hard-core bosons, spin liquids PACS: $75.10 . J m, 05.50 .+q, 05.30 .-d$

\section{INTRODUCTION}

Frustrated quantum magnets are one of the most challenging avatars of the problem of strongly correlated systems [1]. On one hand, the implementation of linear spin wave theory is not straightforward because of the infinite degeneracy of the classical ground state, and it often fails to give a consistent picture because quantum fluctuations destroy magnetic long range order. On the other hand, Quantum Monte Carlo, which has resolved long-standing issues such as the ordered nature of the spin $1 / 2$ Heisenberg model on the square lattice [2], suffers from a very severe minus sign problem in the presence of frustration, and is in most cases of little use.

In view of these difficulties, a standard approach to investigate such systems consists in assuming that there is a small parameter, and to perform an extrapolation of the results derived under this assumption. The parameter assumed to be small can be the inverse number of sites, as for instance in exact diagonalizations of finite clusters $[3,4,5]$, the coupling constants between independent building bricks, as in series expansions [6] or more pedestrian perturbative approaches [7], the inverse number of flavors, as in large$N$ approaches [8], etc... These approaches have reached a high level of sophistication, and they have brought a lot of insight into the properties of several frustrated models. However, they are limited in two respects. First of all, in most cases the parameter assumed to be small is in fact not small, and the reliability of the result is always a matter of debate. This problem is reminiscent of the spin $1 / 2$ Heisenberg model on the square lattice, for which the presence of long-range antiferromagnetic order predicted by linear 
spin wave theory [9] (an expansion in 1/S) has been challenged until Quantum Monte Carlo simulations have confirmed this prediction beyond reasonable doubt [2]. Usually, it takes another, independent approach to prove the correctness of the prediction. This is not too serious however. In fact, the history of solid state physics is paved with examples of theories that were used before one could prove they were correct. But there is another, more important limitation of such approaches: If they are able to signal that a given paradigm (long-range order, dimer order,...) fails, they are usually unable to provide a useful description of the more exotic physics that might take place. For instance, a simple criterion that would allow one to prove or disprove the presence of an RVB (Resonating Valence Bond) spin liquid behavior on the basis of e.g. exact diagonalizations of small clusters in a Heisenberg model is still lacking.

In that respect, significant progress has been made recently in the context of what one may call minimal models. The philosophy of these approaches is very different: The goal is to construct models which contain at least one specific ingredient believed to lead to new physics, while keeping things as simple as possible to allow a satisfactory understanding of the model. As we shall see, such approaches lead to the identification of new paradigms, which can, at least in principle, be tested in the context of more realistic Heisenberg models. In the following, I will discuss two such models. The first one is the so-called Quantum Dimer Model on the triangular lattice, for which the presence of an RVB liquid phase has been firmly established. The second one is a model of interacting bosons with correlated hopping, a model thought to be relevant in the context of frustrated coupled dimers, for which the presence of a phase with pairing but no single particle Bose condensation has been suggested. In both cases, the physical consequences in terms of frustrated magnetic models are radically different from what could be directly identified. The main difficulty of this approach is to convincingly relate the minimal models to realistic situations. For both cases, some recent attempts will be outlined.

\section{QUANTUM DIMER MODELS}

Quantum Dimer Models have been introduced very early after the discovery of high temperature superconductivity in cuprates to describe the spin liquid phase which, according to Anderson's RVB theory [10], should lead to an electronic mechanism of superconductivity upon doping. The Hilbert space of such models consists of nearest-neighbor dimer coverings of a given lattice, and the Hamiltonian that acts in this Hilbert space contains two types of terms: kinetic terms that rearrange the dimers around finite clusters, and potential terms which penalize or favor certain configurations. The simplest version of the model on the square lattice is defined by the Hamiltonian:

$$
H=\sum_{\square}[-t(|\mathbf{=}\rangle\langle\mathbf{I}|+| \mathbf{I}\rangle\langle\mathbf{=}|)+v(|\mathbf{=}\rangle\langle\mathbf{\Xi}|+| \mathbf{I}\rangle\langle\langle\mathbf{I}|)],
$$

where the sum runs over all square plaquettes. The kinetic terms controlled by the amplitude $-t$ change the dimer covering of every flippable plaquette, i.e. of every plaquette containing two dimers facing each other, while the potential terms controlled by the interaction $v$ describe a repulsion $(v>0)$ or an attraction $(v<0)$ between dimers 


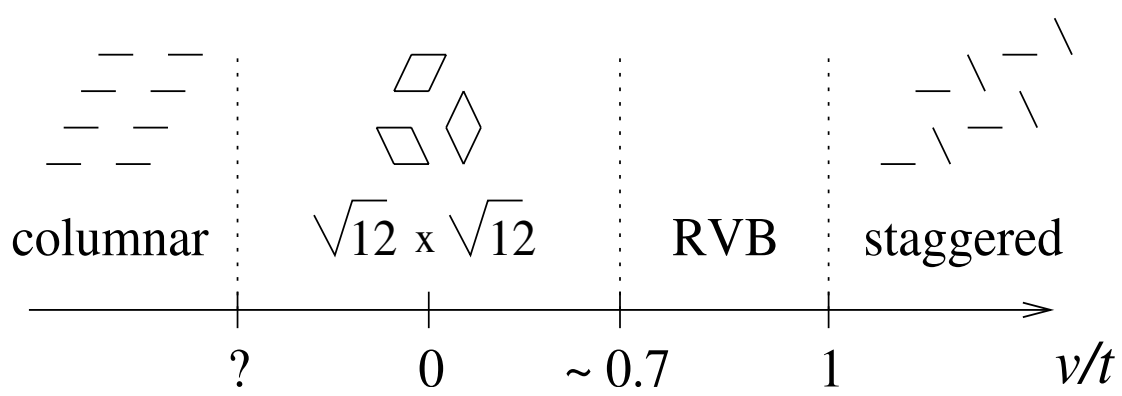

FIGURE 1. Phase diagram of the Quantum Dimer Model on the triangular lattice after Moessner and Sondhi.

facing each other. This model was introduced by Rokhsar and Kivelson [11] who proved that, for $v / t=1$ (the RK point), the sum of all configurations with equal weight is a ground state of the model, reminiscent of Anderson's RVB picture [10]. This is as close as one can get to the RVB physics on the square lattice though. The dimer-dimer correlations at the RK point decay very slowly (as a power law) [12], and away from this point, the model is believed to develop plaquette or dimer long-range valence bond order [13].

The investigation of these models accelerated when Moessner and Sondhi suggested, in 2001, that the same model on the triangular lattice possesses an RVB phase [14]. Their conclusion was based on the observation that the Fourier transform of the dimer-dimer correlations, calculated by a finite temperature Quantum Monte Carlo algorithm after mapping the model on a 3D Ising model, was featureless and temperature independent at low temperature for $(v / t)_{c} \leq v / t \leq 1$ with $(v / t)_{c} \simeq 0.7$. This conclusion was comforted by the observation that the dimer-dimer correlations decay exponentially at the RK point $[15,16]$, leaving the door open for a liquid phase in a finite parameter range. The phase diagram suggested by Moessner and Sondhi on the basis of their finite temperature Quantum Monte Carlo results is sketched in Fig. 1.

While the interpretation in terms of an RVB ground state is very attractive, it is a priori not the only possible explanation. In particular, long-range order involving higher-order dimer correlations could lead to exponentially decaying dimer-dimer correlations. To exclude this possibility and establish firmly the presence of a true liquid phase, one needs a criterion that excludes any kind of long-range order. It turns out that it is indeed possible to come up with such a criterion thanks to a property known as topological degeneracy. This property relies on two observations: First, on the triangular lattice, the parity of the number of dimers intersecting a given closed line is conserved. On a torus, this leads to four topological sectors defined by the parity along two orthogonal diameters of the torus. Second, in a liquid phase, the matrix elements of any local operator between states in different topological sectors vanish in the thermodynamic limit. In addition, the expectation value of any local operator, for instance the Hamiltonian, is expected be independent of the topological sector in the thermodynamic limit. So, if one can access the ground state energies of finite clusters in different topological sectors, they are expected to converge toward the same value when the system size increases. This scaling 


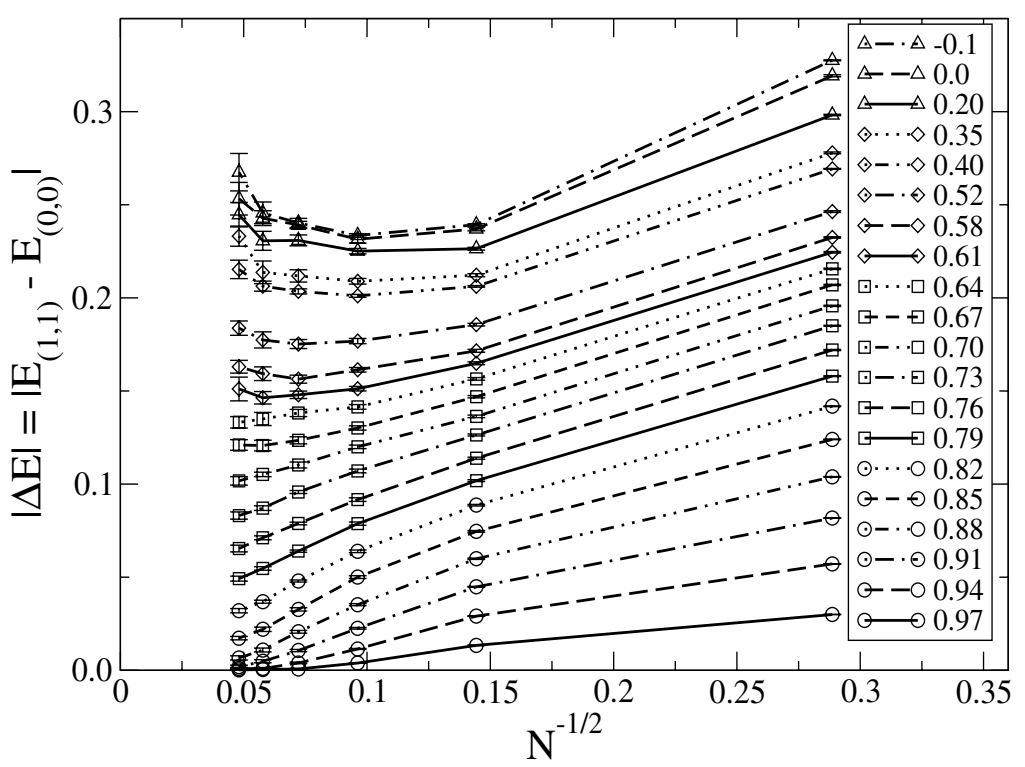

FIGURE 2. Test of topological degeneracy for the Quantum Dimer Model on the triangular lattice: Absolute value of the energy difference between two topological sectors for diamond-like clusters as a function of $1 / \sqrt{N}(N=$ number of sites) for several values of $v / t$. There is a clear change of behavior around $v / t \simeq 0.8$. Lines are guides to the eye.

turned out to be rather subtle, due in particular to the presence of oscillations in the energy difference as a function of the size for square-like clusters, and it is only thanks to a zero-temperature Green's function Quantum Monte Carlo algorithm [17] that large enough clusters (up to more than 500 sites) could be investigated to be able to check for such a scaling [18]. To cut a long story short, it turns out that the energy difference between topological sectors indeed scales to zero in a finite parameter range below the $\mathrm{RK}$ point. This is best seen for diamond-like clusters, for which there is a clear change of scaling behaviour around $v / t=0.8$ (see Fig. 2).

The analysis of the ground state topological sector for smaller values of $v / t$ has allowed one to pin down the value of the transition between the $\sqrt{12} \times \sqrt{12}$ phase and the columnar phase to $v / t \simeq-0.8$, and the calculation of the dimer-dimer correlation function has confirmed the identification of the various phases. So a fair understanding of the model has been reached, as one has the right to expect for a minimal model. The list of open issues includes the nature of the transition between the $\sqrt{12} \times \sqrt{12}$ phase and the RVB phase, and the role of vison excitations in the low-energy properties of the RVB phase [19].

The physical implications of these results are of two kinds. The topological degeneracy is in itself a rather interesting property with potential (if as yet remote) applications for quantum computing [20, 21]. Regarding the properties of actual Mott insulators, these results will constitute the first viable theory of an RVB liquid phase insomuch as one can come up with a reasonably reliable way of mapping realistic models onto QDM. 
This requires some care to be taken. For instance, the original proposal of Rokhsar and Kivelson [11] to truncate the Hilbert space of an SU(2) Heisenberg model on a given lattice to the singlet subspace spanned by nearest-neighbor singlet dimer coverings does not work in general: For the square and triangular lattices it would lead to gapped phases with plaquette or columnar order, whereas it is by now well established that the spin- $1 / 2$ Heisenberg antiferromagnet sustains long range magnetic order on both lattices $[2,3]$. The minimal requirement for such an approach to be relevant is to have some reason to believe that the variational basis spanned by nearest-neighbor singlet dimer coverings on a given lattice is a good one. No general rule could be worked out so far, but the presence of extra degrees of freedom constitutes a promising route. For instance, for the trimerized kagome lattice, which can be thought of as a triangular lattice of triangles, an expansion in the inter-triangle coupling leads to first order to an effective spin-chirality Hamiltonian which lives on a triangular lattice, and which sustains two spin 1/2 degrees of freedom: one for the total spin of each triangle, one for the chirality [7]. A mean-field decoupling of spin and chirality leads to a ground-state manifold made of all dimer coverings of the triangular lattice. For that model, an expansion à la Rokhsar-Kivelson is expected to lead to a relevant QDM. Unfortunately the QDM that can be derived along these lines [22] involves a competition between kinetic terms which leads to a severe minus sign problem, and it is unclear at the moment whether its phase diagram contains an RVB spin liquid phase.

Another, in a certain sense more physical, way to have extra degrees of freedom is to consider Mott insulators with orbital degeneracy, in which case the pseudo-spin describes the choice of orbital for the electrons. In an investigation of the KugelKhomskii [23] model relevant to $\mathrm{LiNiO}_{2}$, a layered spin-1/2 $\mathrm{Ni}$ oxide with twofold orbital degeneracy which resists both orbital and magnetic ordering down to very low temperatures [24], Vernay et al [25] came across a phase where the low energy sector can again be traced back to dimer coverings of the triangular lattice. As for the trimerized kagome, the effective QDM involves a competition between kinetic terms, but this time, the relative sign is such that a Green's function Quantum Monte Carlo algorithm free of any sign problem can be implemented. The problem is still under investigation, but it is already clear that an RVB liquid phase exists in a relevant parameter range [26].

So, to summarize, after ups and downs in the way they were received by the community, Quantum Dimer Models have finally proven to be very useful in identifying a true RVB behavior when the simple Rohksar-Kivelson model is put on the triangular lattice. The implications for Mott insulators are neither obvious nor direct, but there is increasing evidence that the status of QDM might indeed be upgraded from minimal models to effective models when extra-degrees of freedom such as orbital degeneracy are present.

\section{BOSONIC MODELS WITH CORRELATED HOPPING}

The models of interacting bosons (with or without disorder) have been the subject of a very active investigation over the past fifteen years. They are studied for a variety of reasons, coming from different experimental systems, such as Josephson junction arrays [27], ${ }^{4} \mathrm{He}$ in porous media [28], disordered films with superconducting and insulating phases [29], or more recently in the context of atoms trapped in an optical lattice [30]. 


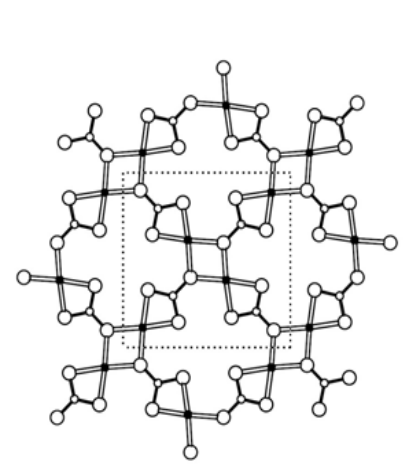

(a)

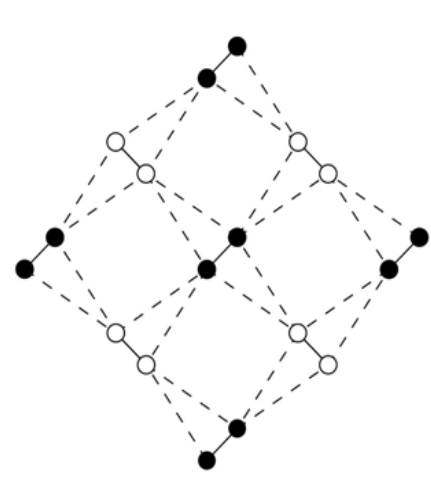

(b)

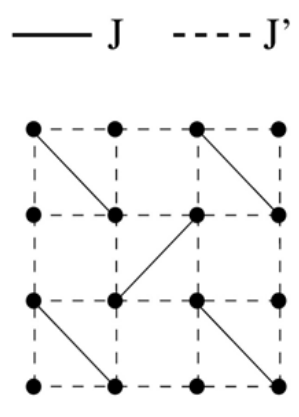

(c)

FIGURE 3. Three representations of a layer of $\mathrm{SrCu}_{2}\left(\mathrm{BO}_{3}\right)_{2}$ : (a) Actual structure of a layer. Solid circles stand for $\mathrm{Cu}$, large open circles for $\mathrm{O}$, small open circles for $\mathrm{B}$; (b) Orthogonal dimer model formed by $\mathrm{Cu}$ atoms inside a layer; (c) Shastry-Sutherland model, a square lattice with coupling $J^{\prime}$ between nearest-neighbors and coupling $J$ between one quarter of the next-nearest neighbors in such of way that it is topologically equivalent to the orthogonal dimer model.

The interplay of interaction, disorder and kinetic energy leads to a ground state that can be a superfluid, a Bose glass, a Mott insulator or a supersolid [31, 32, 33, 34, 35, 36, 37]. In the context of spin models too, the Schwinger boson mean-field theory provides a useful description of magnetism in the bosonic language [38, 39, 40, 41].

Over the last decade, bosons have also been used in the context of quantum magnetism to describe the magnetization process of gapped systems with a singlet ground state such as spin ladders, the triplets induced by the magnetic being treated as hard-core bosons. These bosons may condense, leading to the ordering of the transverse component of the spins, but they might as well undergo a superfluid-insulator transition, leading to magnetization plateaux [42]. For pure SU(2) interactions, and without disorder, the common belief is that the only alternative, not realized so far in quantum magnets, is a supersolid, i.e. a coexistence of these phases.

However, the case of frustrated magnets is (partially at least) outside the scope of standard investigations. Let us consider for instance the case of the orthogonal dimer model realized in $\mathrm{SrCu}_{2}\left(\mathrm{BO}_{3}\right)_{2}$ [43] (see Fig.3). The antiferromagnetic Heisenberg model on this lattice is known as the Shastry-Sutherland model [44]. It has the peculiarity that the product of singlets on diagonal bonds is always an eigenstate, and that it is the ground state if the inter-dimer coupling is not too large. In that case, the spectrum is expected to be gapped, and one might naively expect that elementary excitations simply consist of triplets delocalized on all dimers. The frustration that leads to the exact singlet ground state has another remarkable consequence however. It leads to a drastic reduction of the kinetic energy of a single triplet, which only shows up to sixth order in the ratio of the inter-dimer to intra-dimer coupling, and the major source of kinetic energy of triplets is a correlated hopping term whereby a triplet can hop on a neighbouring site provided there is another triplet nearby, with an amplitude which is only of second order in the 


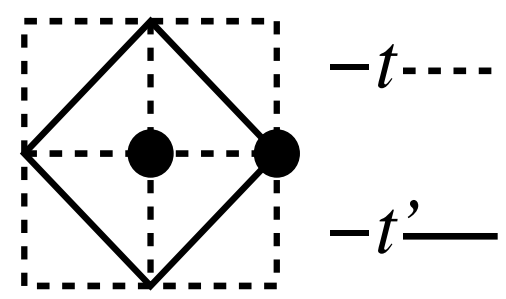

FIGURE 4. The diagonal bonds $\left(-t^{\prime}\right)$ denote a correlated-hopping process, and the nearest neighboring bonds denote the single particle hopping $(-t)$.

ratio of the inter-dimer to intra-dimer coupling [45, 46]. For the two triplet problem, such a correlated hopping term should lead to a bound state, and indeed ESR experiments have revealed the presence of an $S=2$ bound state that should cross the singlet ground state before the first triplet excitations in the presence of a magnetic field. However, to understand the influence of this correlated hopping on the magnetization process of $\mathrm{SrCu}_{2}\left(\mathrm{BO}_{3}\right)_{2}$, one needs to go beyond the two triplet problem and to study the finite density case. It turns out that the physics of such correlated hopping was not investigated in details for bosons.

Before embarking upon a detailed analysis of $\mathrm{SrCu}_{2}\left(\mathrm{BO}_{3}\right)_{2}$, which should in any case contain other terms of the same order, namely antisymmetric inter- and intradimer Dzyaloshinskii-Moriya interactions, it seems wise to turn to minimal models of correlated hopping to see which kind of physics they might induce. The simplest model of this kind lives on a square lattice and is defined by the Hamiltonian:

$$
H=-t \sum_{\mathbf{r}} \sum_{\delta= \pm x, \pm y} b_{\mathbf{r}+\delta}^{\dagger} b_{\mathbf{r}}-\mu \sum_{\mathbf{r}} n_{\mathbf{r}}-t^{\prime} \sum_{\mathbf{r}} \sum_{\delta= \pm x} \sum_{\delta^{\prime}= \pm y} n_{\mathbf{r}}\left\{b_{\mathbf{r}+\delta}^{\dagger} b_{\mathbf{r}+\delta^{\prime}}+h . c .\right\}
$$

where $b_{\mathbf{r}}^{\dagger}, b_{\mathbf{r}}$ are boson operators and $n_{\mathbf{r}}=b_{\mathbf{r}}^{\dagger} b_{\mathbf{r}} . t$ and $t^{\prime}$ are the measures of single particle and correlated-hopping respectively (see Fig. 4).

The basic properties of this model have been derived on the basis of a detailed analysis of the uniform mean-field solution obtained by decoupling the Hamiltonian with the help of the following order parameters:

$$
\begin{aligned}
\Delta & =\left\langle b_{\mathbf{r}}^{\dagger} b_{\mathbf{r} \pm \delta}^{\dagger}\right\rangle \quad \text { (pairing amplitude) } \\
\kappa & =\left\langle b_{\mathbf{r}}^{\dagger} b_{\mathbf{r} \pm \delta}\right\rangle, \kappa^{\prime}=\left\langle b_{\mathbf{r} \pm \delta}^{\dagger} b_{\mathbf{r} \pm \delta^{\prime}}\right\rangle \quad \text { (kinetic amplitudes) } \\
n & =\left\langle b_{\mathbf{r}}^{\dagger} b_{\mathbf{r}}\right\rangle \quad \text { (particle density) }
\end{aligned}
$$

where $\delta \neq \delta^{\prime}$, and $\delta, \delta^{\prime}=x, y$.

Surprisingly enough, the mean-field solution does not comply with the current belief that the ground state of bosonic models should either have single particle Bose-Einstein Condensation (BEC) resulting in a superfluid or supersolid phase, or be insulating: For strong enough correlated hopping, there appears a phase at low density in which 




FIGURE 5. The mean-field quantum phase diagram. $n^{*}$ is the critical density at which, for a given $\alpha=t^{\prime} /\left(t+t^{\prime}\right)$, the single particle condensate density $n_{c}$ becomes zero. This marks the onset of the pairing phase with gapped quasi-particle excitations.

the single particle condensate has disappeared while pairing amplitudes remain finite, leading to an analog of a superconducting phase with low-lying collective excitations (see Fig. 5).

Before discussing further the nature of the solution and the implications for quantum frustrated magnets, let us first comment on the relevance of the uniform mean-field solution of Eq. (2). As is well documented for several bosonic and fermionic models, phase separation is a natural instability that can in particular compete with pairing [47]. The present case is no exception, and preliminary exact diagonalization results for the model of Eq. (2) reveal that a phase separation indeed takes place for large enough $t^{\prime} / t$ in a significant part of the phase diagram. For the physical situation we are interested in, this is not a problem however. Due to the antiferromagnetic exchange present in the original model, triplets experience a repulsion between nearest neighbors and beyond. This repulsion can be estimated from high order perturbation theory, and in $\mathrm{SrCu}_{2}\left(\mathrm{BO}_{3}\right)_{2}$ it is believed to be responsible for the magnetization plateaux at 1/8,1/4 and 1/3. At low density, this repulsion is not expected to lead to a superfluid-insulator transitions: it decays exponentially with distance and can only lead to such a transition for not too high commensurability, hence not too small density. But this repulsion will prevent the system from undergoing a phase separation.

If repulsion supresses phase separation, it could as well supress any tendency towards pairing, and in fact it certainly will if the nearest neighbour repulsion $V_{1}$ is strong enough. So to ascertain the relevance of the physics to be discussed, one should also check that pairing is more robust than phase separation. In the case where there is only pair hopping, the condition for the formation of a bound-state can easily be derived. A pair of nearest neighbour particles will have a potential energy equal to $V_{1}$, and its motion is described by a tight-binding model that lives on the square lattice formed by the bond with amplitude $t^{\prime}$. The minimal energy of such a pair is thus equal to $V_{1}-4 t^{\prime}$. A bound state will thus form as soon as $V_{1}<4 t^{\prime}$, regardless of the value of the longer- 
range repulsion. Preliminary results indicate that phase seperation disappears for much smaller values of $V_{1}$, of the order of $t^{\prime} / 2$. So in a large portion of the parameter space, one expects to find a tendency towards pairing without phase separation.

Finally, the structure of the uniform mean-field solution of a model that includes boson-boson repulsion will be exactly the same as the one for Eq. (2) if the repulsion terms are decoupled in the density-density channel. This will just amount to a redefinition of the chemical potential. So we believe that the simple uniform mean-field solution of the minimal model of Eq. (2) should contain the relevant physics of more complicated models at low doping.

The results of our mean-field calculation are similar to those obtained on a different problem in the context of the atomic gases [48]. These are studies regarding the transition from a purely molecular condensate (MC) to an atomic condensate with a non-zero fraction of the molecular condensate present $(\mathrm{AC}+\mathrm{MC})$ accross the Feshbach resonance. Our pairing phase is like the $\mathrm{MC}$, and the single particle (BEC) phase is analogous to the AC+MC.

Let us now comment further on the physics of the solution. As far as symmetry is concerned, the mean-field Hamiltonian explicitly breaks the U(1) gauge symmetry, but it is still invariant under global $Z_{2}$ symmetry, that is under $b_{\mathbf{r}} \rightarrow-b_{\mathbf{r}}$. In other words, the gauge symmetry $b_{\mathbf{r}}^{\dagger} \rightarrow b_{\mathbf{r}}^{\dagger} e^{i \phi}$ leaves the mean-field Hamiltonian invariant for $\phi=\pi$. This residual Ising-like symmetry will also be broken if there is single particle BEC (because $\langle b\rangle \neq 0$ ). What we have in Fig. (5) is such an Ising symmetry breaking quantum phase transition, where $n_{c}$ is the relevant order parameter. The pairing phase respects this $Z_{2}$ symmetry while the single particle BEC phase breaks it spontaneously. These results suggest that the system should undergo a Kosterlitz-Thouless (KT) transition whatever the density, followed by an Ising transition if $n>n^{*}$.

Finally, let us briefly discuss the physical implications of these results for the magnetization process of gapped quantum magnets. According to the discussion of the thermodynamics in the boson language, we expect to observe a KT transition for any magnetization, followed by an Ising transition if the magnetization is larger than a critical value. This will remain essentially true for 3D systems, the KT transition being replaced by a true phase transition toward an ordered phase. However, we also expect very significant differences between the zero temperature phases. Single particle BEC means magnetic long-range order, and the system is expected to have gapless transverse spin waves. In other words, the gap detected in spectroscopies such as inelastic neutron scattering or NMR will vanish. However, at low magnetization, we only have pair BEC. The order implied by this pair BEC will be of nematic type since the transverse components of the spins within a pair can be flipped without changing the correlations. But more importantly, there is a gap to single particle excitations, i.e. to single spin flips. Although the system is gapless in this phase, we thus expect to observe a gap in neutron scattering or NMR, the gapless excitations appearing only in the channel $\Delta S=2$.

To check the mean-field predictions, it would of course be desirable to perform well controlled numerical simulations on the model of Eq. (2). It turns out that, at least in principle, this is possible: The model of Eq. (2) can be simulated both at zero temperature and at finite temperature by Quantum Monte Carlo algorithms free of any minus sign problem. Work is in progress along these lines. 


\section{CONCLUSIONS}

After nearly twenty years of hard work on frustrated quantum magnets following the discovery of high temperature superconductivity in cuprates, the belief that frustration can induce properties radically different from those of their unfrustrated cousins is shared by many researchers in the field. It has proven extremely difficult however to come up with reliable results showing for instance that an exotic ground state such as a Resonating Valence Bond spin liquid is realized starting from the Heisenberg model on a frustrated lattice. In that respect, minimal models such as those presented in this paper are very promising. They are in particular extremely helpful in reliably identifying and describing new exotic phases of quantum magnets. The price to pay is of course that, unlike the effective models derived by perturbation expansion or more sophisticated numerical methods, these models, which are desgined to be simple and 'soluble', are not directly related to actual systems. But to bridge this gap is a much better defined program than looking for exotic properties directly on realistic models of quantum magnets. In that respect, the results obtained in the context of the Kugel-Khomskii model of orbitally degenerate Mott insulators are very encouraging. With the rapidly growing family of frustrated magnetic insulators, one may thus hope without being too optimistic that some of the exotic physics found in these and other minimal models will soon be observed in experiments.

\section{ACKNOWLEDGMENTS}

It is a pleasure to thank all the colleagues with whom I have had a chance to work on these and related issues over the years, in particular Federico Becca, Rachel Bendjama, Julien Dorier, Patrik Fazekas, Michel Ferrero, Dima Ivanov, Brijesh Kumar, Andreas Läuchli, Matthieu Mambrini, Shin Miyahara, Karlo Penc, Didier Poilblanc, Arnaud Ralko and François Vernay. This work was supported by the Swiss National Fund and by MaNEP.

\section{REFERENCES}

1. See e.g. the Proceedings of the Highly Frustrated Magnetism 2003 Conference (Grenoble, France, 26-30 August 2003), J. Phys.: Condens. Matter 16 (2004), and references therein.

2. J. D. Reger and A. P. Young, Phys. Rev. B 37, 5978 (1988).

3. B. Bernu, C. Lhuillier et L. Pierre, Phys. Rev. Lett. 69, 2590 (1992).

4. H. J. Schulz, T. A. L. Ziman, D. Poilblanc, J. Physique I 6, 675 (1996).

5. P. Lecheminant, B. Bernu, C. Lhuillier, L. Pierre and P. Sindzingre, Phys. Rev. B 56, 2521 (1997).

6. $\quad$ M. P. Gelfand, Phys. Rev. B 42, 8206-8213 (1990).

7. F. Mila, Phys. Rev. Lett. 81, 2356 (1998).

8. $\quad$ N. Read and S. Sachdev, Phys. Rev. Lett. 62, 1694-1697 (1989).

9. P. W. Anderson, Phys. Rev. 86, 694-701 (1952).

10. P. W. Anderson, Mater. Res. Bull. 8, 153 (1973); P. W. Anderson, Science 235, 1196 (1987).

11. D.S. Rokhsar and S.A. Kivelson, Phys. Rev. Lett. 61, 2376 (1988

12. M. E. Fisher and J. Stephenson, Phys. Rev. 132, 1411 (1963).

13. P. W. Leung, K. C. Chiu, and K. J. Runge, Phys. Rev. B 54, 12938-12945 (1996).

14. R. Moessner and S.L. Sondhi, Phys. Rev. Lett. 86, 1881 (2001). 
15. P. Fendley, R. Moessner, and S.L. Sondhi, Phys. Rev.B 66, 214513 (2002).

16. A. Ioselevich, D.A. Ivanov, and M.V. Feigel'man, Phys. Rev.B 66, 174405 (2002).

17. Nandini Trivedi and D. M. Ceperley, Phys. Rev.B 41, 4552 (1990); M. Calandra and S. Sorella, Phys. Rev.B 57, 11446 (1998).

18. A. Ralko, M. Ferrero, F. Becca, D. Ivanov, and F. Mila Phys. Rev.B 71, 224109 (2005).

19. D.A. Ivanov, Phys. Rev.B 70, 094430 (2004).

20. L.B. Ioffe, M.V. Feigel'man, A. Ioselevich, D. Ivanov, M. Troyer, and G. Blatter, Nature 415, 503 (2002).

21. G. Misguich, V. Pasquier, F. Mila, and C. Lhuillier, Phys. Rev. B 71, 184424 (2005).

22. M. E. Zhitomirsky, Phys. Rev. B 71, 214413 (2005).

23. K. I. Kugel et D. I. Khomskii, Usp. Fiz. Nauk 136, 621 (1982) [Sov. Phys. Usp. 25, 231 (1982)].

24. See F. Reynaud, D. Mertz, F. Celestini, J.-M. Debierre, A. M. Ghorayeb, P. Simon, A. Stepanov, J. Voiron, and C. Delmas Phys. Rev. Lett. 86, 3638-3641 (2001), and references therein.

25. F. Vernay, K. Penc, P. Fazekas, and F. Mila, Phys. Rev. B 70, 014428 (2004).

26. F. Vernay, F. Becca, A. Ralko, F. Mila, unpublished.

27. L. J. Geerligs, M. Peters, L. E. M. de Groot, A. Verbruggen, and J. E. Mooij, Phys. Rev. Lett. 63, 326 (1989).

28. M. H. W. Chan, K. I. Blum, S. Q. Murphy, G. K. S. Wong, and J. D. Reppy, Phys. Rev. Lett. 61, 1950 (1988).

29. D. B. Haviland, Y. Liu, and A. M. Goldman, Phys. Rev. Lett. 62, 2180 (1989).

30. M. Greiner, O. Mandel, T. Esslinger, T. W. Hansch, and I. Bloch, Nature (London) 415, 39 (2002).

31. P. B. Weichman, G. Grinstein, and D. S. Fisher, Phys. Rev. B 40, 546 (1989).

32. T. Klien, I. Joumard, S. Blanchard, J. Marcus, R. Cubitt, T. Giamarchi, and P. L. Doussal, Nature 413, 404 (2001).

33. F. Alet and E. S. Sorensen, Phys. Rev. B 70, 024513 (2004).

34. J. K. Freericks and H. Monien, Phys. Rev. B 53, 2691 (1996).

35. K. Sheshadri, H. R. Krishnamurthy, R. Pandit, and T. V. Ramakrishnan, Phys. Rev. Lett. 75, 4075 (1995).

36. P. Sengupta, L. P. Pryadko, F. Alet, M. Troyer, and G. Schmid, Phys. Rev. Lett. 94, 207202 (2005).

37. A. van Otterlo and K.-H. Wagenblast, Phys. Rev. Lett. 72, 3598 (1994).

38. D. P. Arovas and A. Auerbach, Phys. Rev. B 38, 316 (1988).

39. S. Sarkar, C. Jayaprakash, H. R. Krishnamurthy, and M. Ma, Phys. Rev. B 40, 5028 (1989).

40. P. Chandra, P. Coleman, and A. I. Larkin, J. Phys.: Condens. Matter 2, 7933 (1990).

41. F. Mila, D. Poilblanc, and C. Bruder, Phys. Rev. B 43, 7891 (1991).

42. T. M. Rice, Science 298, 760 (2002), and see references therein.

43. H. Kageyama, K. Yoshimura, R. Stern, N. V. Mushnikov, K. Onizuka, M. Kato, K. Kosuge, C. P. Slichter, T. Goto, and Y. Ueda, Phys. Rev. Lett. 82, 3168 (1999).

44. B. Shastry and B. Sutherland, Physica B 108, 1069 (1981).

45. T. Momoi and K. Totsuka, Phys. Rev. B 62, 15067 (2000).

46. See S. Miyahara and K. Ueda, J. Phys.: Condens. Matter 15, R327 (2003), and references therein.

47. See e.g. V. Rousseau, G. G. Batrouni, and R. T. Scalettar, Phys. Rev. Lett. 93, 110404 (2004), and references therein.

48. M. W. H. Romans, R. A. Duine, S. Sachdev, and H. T. C. Stoof, Phys. Rev. Lett. 93, 020405 (2004). 